\title{
ON THE FORM OF THE SPITZER LEAVITT LAW AND ITS DEPENDENCE ON METALLICITY
}

\author{
D. Majaess ${ }^{1}$, D. G. Turner ${ }^{1}$, ANd W. Gieren ${ }^{2}$ \\ ${ }^{1}$ Department of Astronomy \& Physics, Saint Mary's University, Halifax, NS B3H 3C3, Canada; dmajaess@ap.smu.ca \\ ${ }^{2}$ Departamento de Astronomía, Universidad de Concepción, Casilla 160-C, Concepción, Chile \\ Received 2013 April 5; accepted 2013 May 30; published 2013 July 16
}

\begin{abstract}
The form and metallicity dependence of Spitzer mid-infrared Cepheid relations are a source of debate. Consequently, Spitzer 3.6 and $4.5 \mu \mathrm{m}$ period-magnitude and period-color diagrams were re-examined via robust routines, thus providing an alternative interpretation to consider. The relations (nearly mean-magnitude) appear nonlinear over an extensive baseline $\left(0.45<\log P_{0}<2.0\right)$, particularly the period-color trend, which to first order follows constant (3.6-4.5) color for shorter-period Cepheids and may transition into a bluer convex trough at longer periods. The period-magnitude functions can be described by polynomials $(\mathrm{e} . \mathrm{g}$., $[3.6 \mu \mathrm{m}]=$ $\left.K_{0}-(3.071 \pm 0.059) \log P_{0}-(0.120 \pm 0.032) \log P_{0}^{2}\right)$, and Cepheid distances computed using 3.6 and $4.5 \mu \mathrm{m}$ relations agree with each other and the latter provides a first-order consistency check (CO sampled at $4.5 \mu \mathrm{m} \mathrm{does} \mathrm{not}$ seriously compromise those distances). The period-magnitude relations appear relatively insensitive to metallicity variations $\left([\mathrm{Fe} / \mathrm{H}] \sim 0\right.$ to $\left.-0.75|\gamma|<0.1 \mathrm{mag} \mathrm{dex}^{-1}\right)$, a conclusion inferred partly from comparing galaxy distances established from those relations and NED-D $(n>700)$, yet a solid conclusion awaits comprehensive mid-infrared observations for metal-poor Cepheids in IC $1613([\mathrm{Fe} / \mathrm{H}] \sim-1)$. The Cepheid-based distances were corrected for dust obscuration using a new ratio (i.e., $A_{3.6} / E_{B-V}=0.18 \pm 0.06$ ) deduced from GLIMPSE (Spitzer) data.
\end{abstract}

Key word: stars: variables: Cepheids

Online-only material: color figures

\section{INTRODUCTION}

Spitzer mid-infrared observations of Cepheid variables have been employed to constrain stellar mass loss and anchor the cosmic distance scale (e.g., Neilson et al. 2009; Monson et al. 2012). Regarding the latter, the principal aim is to establish firmer constraints on the Hubble constant and cosmological models (Freedman et al. 2011). However, ambiguities linger concerning the form and metallicity dependence of mid-infrared Cepheid relations.

The impetus for obtaining Spitzer observations stems partly ${ }^{3}$ from the diminished impact of dust extinction on infraredbased distances $\left(A_{3.6} / A_{V} \sim 0.06\right.$, Section 2.4$)$. Crucially, uncertainties tied to neglecting variations in the extinction law are reduced. The extinction law varies throughout the Galaxy, and a $20 \%$ uncertainty $\left(\sigma_{R_{V}} / R_{V} \sim 0.2\right)$ merely contributes $\sigma_{\mu_{0}} \sim 0$. 01 to the mid-infrared distance modulus (assuming $\left.E_{B-V}=0.10 \pm 0.03\right)$. Conversely, variations in the extinction law can significantly impact Cepheid and star cluster distances that rely solely on shorter-wavelength data (e.g., Turner 2012a; Carraro et al. 2013 and discussion therein).

The aforementioned points reiterate the importance of Spitzer observations. However, a partial account provided below highlights the diverse opinions expressed concerning the form and metallicity dependence of mid-infrared Cepheid relations. Ngeow \& Kanbur (2008), Madore et al. (2009), and Marengo et al. (2010) cited different slopes for linear Spitzer period-magnitude relations, while Neilson et al. (2009) suggested that function is non-linear (see also Ngeow \& Kanbur 2010). For example, Ngeow \& Kanbur (2008) obtained $\alpha=$ -3.26 for the slope of the $3.6 \mu \mathrm{m}$ relation using the OGLE

\footnotetext{
3 See Freedman et al. (2011, their Section 2).

4 Optical Gravitational Lensing Experiment (e.g., Soszyñski et al. 2008, 2010).
}

and $\mathrm{SAGE}^{5}$ data sets $(0.5<\log P<1.7)$, whereas Madore et al. (2009) favored a steeper slope inferred by correlating the Persson et al. (2004) and SAGE catalogs $(\log P>0.8)$. A shallower slope may arise partly from the inclusion of shorter-period Cepheids that could suffer increased photometric contamination, particularly for distant targets (e.g., Macri et al. 2006; Madore et al. 2009; Majaess et al. 2013). However, Ngeow \& Kanbur (2010) deduced analogous period-magnitude relations for relatively nearby Cepheids in the Small Magellanic Cloud (SMC) that occupy low- and high-density regions.

Marengo et al. (2010) and Ngeow et al. (2012b) found certain models produced Spitzer period-magnitude and period-color relations that did not match observations spanning an extended abundance baseline (see also Ngeow et al. 2012a and discussions therein). Observations imply that the same slope characterizes 3.6 and $4.5 \mu \mathrm{m}$ period-magnitude relations for SMC and LMC Cepheids (Ngeow \& Kanbur 2010), which exhibit mean abundances of $[\mathrm{Fe} / \mathrm{H}] \sim-0.75,-0.33$ accordingly (Luck et al. 1998; Mottini et al. 2006). Marengo et al. (2010) attempted to extend the baseline to include Galactic Cepheids $([\mathrm{Fe} / \mathrm{H}] \sim 0)$, but the effort was marred by inconsistencies associated with the available calibrating data (see also Ngeow \& Kanbur 2010 and discussions therein). Hackwell \& Gehrz (1974) and Marengo et al. (2010) noted that the $4.5 \mu \mathrm{m}$ passband samples CO absorption features, which presumably alter the Spitzer Cepheid relations and introduce a metallicity effect. Scowcroft et al. (2011) confirmed the former finding by demonstrating that cooler longperiod Cepheids exhibit colors that are modulated by the absorption and disassociation of $\mathrm{CO}$, whereas their hotter short-period counterparts are less affected since the molecule is gradually disassociated. Freedman \& Madore (2011) estimated that 3.6

\footnotetext{
5 Surveying the Agents of a Galaxy's Evolution (Meixner et al. 2006; Gordon et al. 2011).
} 
and $4.5 \mu \mathrm{m}$ Cepheid data exhibit a metallicity dependence of $\gamma=-0.39 \pm 0.16$ and $-0.25 \pm 0.18 \mathrm{mag} \mathrm{dex}^{-1}$, respectively. The former estimate was revised by Freedman et al. (2011) to $\gamma=-0.09 \pm 0.29 \mathrm{mag} \mathrm{dex}^{-1}$. Those results are tied partly to published abundance estimates for individual LMC Cepheids, yet it is unclear whether systematic effects pervade such individual determinations, ${ }^{6}$ although a mean metallicity derived from an entire sample appears reliable (Luck et al. 1998; Romaniello et al. 2008).

In this study, the form and metallicity dependence of Spitzer period-magnitude and period-color relations are reinvestigated. The relation linking the total extinction at $3.6 \mu \mathrm{m}$ $\left(A_{3.6}\right)$ to the optical color excess $\left(E_{B-V}\right)$ is likewise derived from Spitzer data, as the correlation is necessary for computing Cepheid distances using the period-magnitude relations derived.

\section{ANALYSIS}

\subsection{6 and 4.5 $\mu \mathrm{m}$ Period-Magnitude Relations}

3.6 and $4.5 \mu \mathrm{m}$ period-magnitude relations are now inferred from LMC and SMC Cepheids. The LMC sample was assembled by correlating $\mathrm{HV}^{7}$ Cepheids tabulated by Madore (1985) and Persson et al. (2004) with SAGE (Spitzer) photometry (a mean of random-phase data). Fundamental-mode Cepheids cataloged by OGLE were likewise added to the sample. For the SMC analysis, the Madore (1985) and OGLE catalogs were paired with SAGE photometry. A period cutoff of $\log P_{0}>0.45$ was imposed to mitigate biases stemming from a reputed change in slope near $\log P \sim 0.4$ (Ngeow \& Kanbur 2010), to ensure magnitude uncertainties were satisfactory, to reduce the effects of photometric contamination, and to ensure that brighter Cepheids on the blue edge of the instability strip were not preferentially sampled (Sandage 1988; see also Appendix A in Freedman et al. 2001). The $\log P<2.0$ limit was adopted to avoid the inclusion of Leavitt variables (Grieve et al. 1985), which may adhere to a separate slope (Persson et al. 2004, their Figure 3).

The nearly mean-magnitude period-color (3.6-4.5) relation examined in Section 2.3 is characterized by a higherorder polynomial over the baseline examined, thus hinting that the period-magnitude relations are non-linear. A single linear period-magnitude function does not adequately describe both the short- and long-period domains (see also Neilson et al. 2009). Longer-period Cepheids $(\log P>1.45)$ displayed a $\Delta[3.6 \mu \mathrm{m}] \gtrsim 0$. 06 offset relative to a linear fit, whereby the latter fit yields values systematically too faint. Longer-period Cepheids are important granted the stars are preferentially sampled within remote galaxies in the Hubble flow. Efforts toward achieving precision cosmology $\left(\sigma_{H_{0}} / H_{0}<3 \%\right)$ hinge on reducing sources of uncertainty for such stars, especially since certain Cepheid-based determinations of $H_{0}$ exhibit tension with the new Planck results (Planck Collaboration et al. 2013; see also Freedman et al. 2012; Tammann \& Reindl 2012 and discussions therein).

\footnotetext{
6 The Freedman \& Madore (2011) analysis, which relies on published abundance estimates found in the literature for individual Cepheids, implies that the $B V$ Wesenheit function displays a nearly negligible dependence on metallicity, whereas certain researchers suggest otherwise (Tammann et al. 2003; Majaess et al. 2009; Bono et al. 2010; see also the discussion in Freedman \& Madore 2011 concerning the $\mathrm{VI}_{\mathrm{c}}$ Wesenheit function).

7 Harvard Variable.
}

Robust fitting routines were employed since the method of least squares is acutely sensitive to outliers, ${ }^{8}$ whereas the former techniques are less affected by spurious data and more apt to identify the underlying trend. The robust routine applied seeks to minimize the sum of the absolute deviations, rather than the sum of the squared deviations. The routine was used in concert with a $4 \sigma$ clip, twice iterated. That conservative procedure was followed because Madore et al. (2009) suggested that differences between the various determinations for the period-magnitude relation may arise partly from aggressive clipping/iterations.

The following functional forms were deduced and characterized LMC Cepheids across an extensive baseline $(0.45<$ $\left.\log P_{0}<2.0\right)$ :

$$
\begin{aligned}
{[3.6 \mu \mathrm{m}]=} & (15.917 \pm 0.026)-(3.077 \pm 0.064) \log P_{0} \\
& -(0.114 \pm 0.036) \log P_{0}^{2} \\
{[4.5 \mu \mathrm{m}]=} & (15.890 \pm 0.026)-(3.084 \pm 0.065) \log P_{0} \\
& -(0.082 \pm 0.036) \log P_{0}{ }^{2}
\end{aligned}
$$

For SMC Cepheids the corresponding relations are

$$
\begin{aligned}
{[3.6 \mu \mathrm{m}]=} & (16.411 \pm 0.063)-(3.041 \pm 0.143) \log P_{0} \\
& -(0.145 \pm 0.074) \log P_{0}{ }^{2} \\
{[4.5 \mu \mathrm{m}]=} & (16.382 \pm 0.061)-(3.076 \pm 0.140) \log P_{0} \\
& -(0.104 \pm 0.072) \log P_{0}{ }^{2}
\end{aligned}
$$

The magnitude of the squared term increases slightly if longerperiod Cepheids are given more weight, and the uncertainties subsequently diminish. Yet a single quadratic expression is preferred over two linear relations since the statistical weight of the entire Cepheid demographic can be exploited to reduce uncertainties. Relatively nearby short-period Cepheids detected in the Magellanic Clouds outnumber their long-period counterparts, as low-mass metal-poor stars cross the instability strip with an extended blue loop (Becker 1985). The initial mass function implies that low-mass objects are more numerous, which in concert with the aforementioned trend explains the shift in the distribution of Magellanic Cloud Cepheids toward shorter periods. ${ }^{9}$

\subsection{Metallicity Effects at 3.6 and $4.6 \mu \mathrm{m}$}

The coefficients tied to the pulsation period in Equations (1) and (2) agree to within the uncertainties. Thus the slopes for the 3.6 and $4.5 \mu \mathrm{m}$ period-magnitude relations are comparatively insensitive to metallicity variations from $[\mathrm{Fe} / \mathrm{H}] \sim-0.33$ to -0.75 (see also Ngeow \& Kanbur 2010). The 3.6 and $4.5 \mu \mathrm{m}$ zero points and coefficients for a given Magellanic Cloud agree to within the uncertainties, thus indicating that distances established from the latter passband can serve as a first-order consistency check (CO is sampled in the $4.5 \mu \mathrm{m}$ passband, e.g., Hackwell \& Gehrz 1974).

The impact of metallicity can likewise be assessed by comparing NED-D ${ }^{10}$ and Spitzer-based Cepheid distances. Relative offsets between the SMC/LMC, and the SMC/Milky Way, are now evaluated. The latter comparison provides a desirably

\footnotetext{
8 Certain outliers may be Cepheids exhibiting high mass loss (Neilson et al. 2009).

9 There exists a period-mass-luminosity relation (e.g., Turner 2012b).

10 NASA/IPAC Extragalactic Database Master List of Galaxy Distances (Steer \& Madore 2011).
} 
large abundance baseline $(\Delta[\mathrm{Fe} / \mathrm{H}] \sim-0.75)$. Cepheid distances were calculated via the following relations derived from a weighted mean of Equations (1) and (2):

$$
\begin{aligned}
{[3.6 \mu \mathrm{m}]=} & K_{0}-(3.071 \pm 0.059) \log P_{0} \\
& -(0.120 \pm 0.032) \log P_{0}{ }^{2} \\
{[4.5 \mu \mathrm{m}]=} & K_{0}-(3.083 \pm 0.059) \log P_{0} \\
& -(0.086 \pm 0.032) \log P_{0}{ }^{2}
\end{aligned}
$$

The offset between Cepheids in the SMC/LMC is $\Delta K_{0,3.6}=$ $0.501 \pm 0.068$, while the differential for the SMC/Galaxy is $\Delta K_{0,3.6}=18.938 \pm 0.077$. Marginal extinction corrections were applied to the Cepheid distances (Section 2.4), and the Galactic calibration used is discussed below. The corresponding $4.5 \mu \mathrm{m}$ results are $\Delta K_{0,4.5}=0.480 \pm 0.066$ and $18.921 \pm 0.075$, respectively. The values compare favorably to means tabulated from NED-D data $(n>700)$, namely $\Delta K_{0}=0.450 \pm 0.012$ and $18.930 \pm 0.011$. The results imply values of $\gamma_{3.6} \sim-0.10 \pm 0.10,-0.01 \pm 0.06$ and $\gamma_{4.5} \sim$ $-0.06 \pm 0.10,0.01 \pm 0.06 \mathrm{mag} \mathrm{dex}^{-1}$. The associated uncertainties underscore the need for continued research, and formal uncertainties cited throughout the analysis may underestimate the true uncertainties.

In sum, the analyses imply that distances inferred from the 3.6 and $4.5 \mu \mathrm{m}$ period-magnitude relations are relatively insensitive to variations in metallicity $([\mathrm{Fe} / \mathrm{H}] \sim 0$ to -0.75$)$. However, a comparison including IC 1613 is required since Cepheids in that galaxy $([\mathrm{Fe} / \mathrm{H}] \sim-1)$ are more metal-poor than their SMC counterparts. Reliable Spitzer data are merely readily available for five $\log P<2$ Cepheids in IC 1613 (Freedman et al. 2009), and a comparison at present may be premature given those small statistics, the potential for inadequate instability strip filling, and concerns regarding photometric contamination expressed in the literature (Freedman et al. 2009). Freedman et al. (2011, their Table 1) plan to obtain comprehensive mid-infrared observations for additional Cepheids in IC 1613.

The Galactic mid-infrared data were adopted verbatim from Monson et al. (2012), who presented complete light curves for numerous Galactic Cepheids. Monson et al. (2012) paired their new photometry with IRSB, ${ }^{11}$ parallax, and cluster Cepheid data to establish a Galactic calibration (e.g., Benedict et al. 2007; Fouqué et al. 2007; Turner 2010). That calibration consists of Cepheids with near solar abundances $([\mathrm{Fe} / \mathrm{H}] \sim 0)$, and is hence important for assessing the impact of metallicity. However, the Galactic calibration may presently be the most difficult to construct, since the constituent Cepheids exhibit sizable differential reddening and do not lie at a common distance. The Galactic calibration currently suffers from a lack of solid long-period calibrators, and parameters for cluster Cepheids are being contested and revised (e.g., Anderson et al. 2012; Majaess et al. 2012). The IRSB parameters for long-period Cepheids rely directly on the $p$-factor, the form of which is actively debated given its importance (Gieren et al. 2005; Storm et al. 2011; Ngeow et al. 2012c; Neilson et al. 2012). ${ }^{12}$ Nevertheless, the resulting non-linear fits (Equation (3)) to the Monson et al. (2012) data ${ }^{13}$ yield $K_{0,3.6}=-2.545 \pm 0.045$

\footnotetext{
11 Infrared Surface Brightness technique (Fouque \& Gieren 1997).

$12 \mathrm{~W}$. Gieren et al. (2013, in preparation) aim to constrain the $p$-factor using LMC Cepheids in double-lined eclipsing binary systems (Pietrzyński et al. 2010, 2011).

13 The absolute magnitudes used were an averaged subset from IRSB, cluster Cepheid, and the Benedict et al. (2007) parallax results (see Monson et al. 2012).
}

and $K_{0,4.5}=-2.551 \pm 0.043$. The results were derived by adopting the $\log P_{0}$ coefficient established from Magellanic Cloud Cepheids (Equation (3)), while permitting $\log P_{0}^{2}$ and $K_{0}$ to vary. The aim was to examine whether the resulting coefficients for $\log P_{0}^{2}$ match those determined for Magellanic Cloud Cepheids (Equation (3)). The results agree to first order, namely, $-0.10(3.6 \mu \mathrm{m})$ and $-0.05(4.5 \mu \mathrm{m})$. However, those determinations are merely suggestive owing to the lack of numerous solid long-period calibrators.

\subsubsection{Comparison of the LMC Data}

The Madore et al. (2009) LMC sample results from a correlation of the Persson et al. (2004) and SAGE catalogs (a mean of random-phase data), whereas Scowcroft et al. (2011) subsequently obtained complete mid-infrared light-curve coverage for the Cepheids as part of the Carnegie Hubble Project. Equation (3) is now applied to the LMC data of Madore et al. (2009) and Scowcroft et al. (2011). The period coefficients remained fixed, and the zero point was allowed to vary. Zero points of $K_{0,3.6}=15.939 \pm 0.030$ and $15.911 \pm 0.012$ were derived accordingly. The results agree to within the formal uncertainties, and a visual inspection reveals a reliable fit (Figure 1). A least-squares solution was obtained since extreme outliers are absent.

The Scowcroft et al. (2011) data (warm Spitzer) are tied to a separate photometric reduction, whereas the Madore et al. (2009), Ngeow \& Kanbur (2008), and Figure 1 samples rely on SAGE photometry. A comparison between the SAGE and Scowcroft et al. (2011, hereafter S11) $3.6 \mu \mathrm{m}$ photometry reveals a color offset $\left(\Delta[3.6]_{\text {S11-SAGE }} \sim-0.9 \times(3.6-4.5)_{\text {SAGE }}\right)$. The offset may stem from uncertainties in the photometric standardization, but additional research is ultimately needed to identify the source.

\subsection{Period-Color Relation}

Period-color data for LMC and SMC Cepheids were examined (Figure 1). The observations are characterized by a non-linear fit over the period baseline, whereby shorter-period Cepheids adhere to a near constant color and a convex trough may describe the longer-period domain. ${ }^{14}$ Those trends can be explained in part by CO (see also Hackwell \& Gehrz 1974; Marengo et al. 2010; Scowcroft et al. 2011; Monson et al. 2012). Figure 1 indicates that for shorter-period Cepheids the color is nearly constant as the temperature is sufficiently high to disassociate most CO. For longer-period (cooler) Cepheids CO absorption occurs, and the molecule is gradually disassociated in increasing amounts near the pulsation phase corresponding to temperature maximum. The period-color trend exhibits inflection points near $\log P \sim 0.75, \log P \sim 1.75$, and potentially $\log P>2.2$. Additional analyses are warranted.

A mean color inferred from shorter-period Cepheids near the constant part of the trend yields $(3.6-4.5)=0.023,0.039$ for the LMC and SMC, respectively. The optical to mid-infrared color-excess ratio derived in Section 2.4 implies intrinsic colors of $(3.6-4.5)_{0}=0.015,0.033$ (LMC/SMC). Galactic Cepheids (Monson et al. 2012) exhibit (3.6-4.5) $0=-0.022$, as deduced from a visual match to intermediate-period Cepheids in the LMC. That procedure was followed as the Spitzer Galactic sample does not presently contain numerous shorter-period

\footnotetext{
14 The period-color relation is nearly constant across the entire period baseline when sampled at the hottest pulsation phase (Monson et al. 2012, their Figure 9).
} 


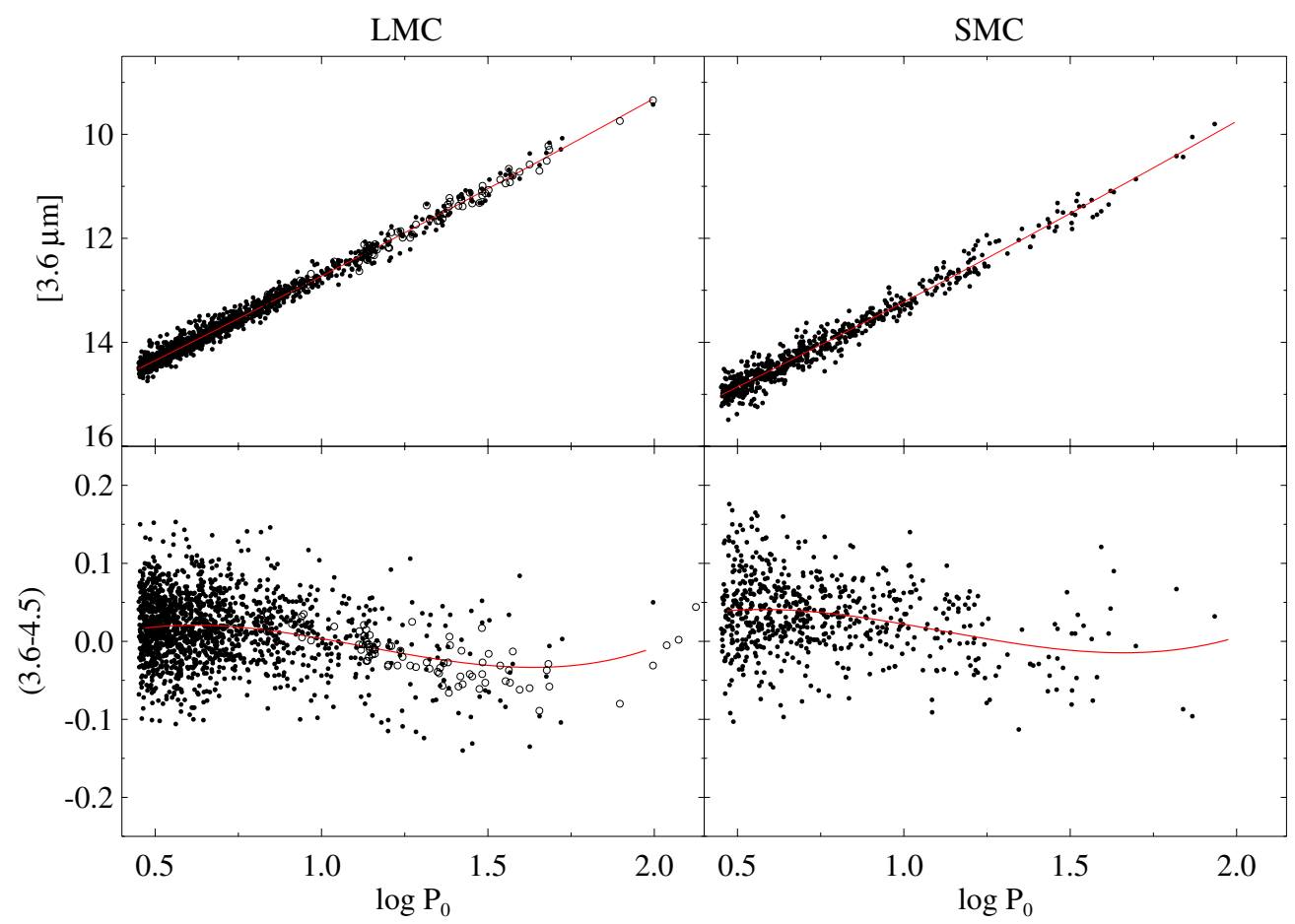

Figure 1. Spitzer period-magnitude and period-color diagrams for Cepheids in the Magellanic Clouds. The (nearly mean-magnitude) data are described by non-linear trends over an extended baseline $\left(0.45<\log P_{0}<2.0\right)$, particularly the period-color relation. Solid lines convey mean fits, and the data shown were cleaned of outliers via an iterative $\sigma$-clip. Filled circles represent SAGE photometry, while open circles are observations from Scowcroft et al. (2011).

(A color version of this figure is available in the online journal.)

Cepheids, and their longer-period counterparts can exhibit rather uncertain reddening estimates. ${ }^{15}$

Offsets between the intrinsic colors of the three galaxies are likely insignificant owing to uncertainties arising from photometric reductions and extinction corrections. Monson et al. (2012) note that the systematic error associated with Spitzer photometry for Galactic Cepheids may be $\sim 0$. 016 in each passband (3.6 and $4.5 \mu \mathrm{m})$, and a similar uncertainty exists for the Magellanic Cloud photometry. More sizable photometric offsets can exist in optical surveys of star clusters (Stetson et al. 2004, their Table 3 for NGC 188) and the Magellanic Clouds (D. Majaess et al., in preparation). Alternatively, comprehensive mid-infrared observations of Cepheids in IC 1613 will dictate whether metallicity offsets the period-color relation $\left(\gamma_{3.6-4.5} \sim\right.$ $\left.-0.07 \mathrm{mag} \mathrm{dex}^{-1}+\ldots\right)$, as metal-poor Cepheids in IC 1613 are predicted to be intrinsically redder than their SMC, LMC, and Galactic counterparts (Monson et al. 2012, their Figure 10).

\subsection{Extinction Law}

Spitzer period-magnitude relations provide Cepheid distances once corrected for dust extinction. Most extragalactic Cepheids observed for the Hubble Space Telescope key project to measure $H_{0}$ are reddened by $E_{B-V} \sim 0.1$ (e.g., Figure 6 in Majaess 2010), and thus the resulting mid-infrared extinction is marginal. There are exceptions such as the Cepheids in NGC 6822 (Gieren et al. 2006) and Centaurus A (Ferrarese et al. 2007), that exhibit comparatively larger reddenings.

The ratios $A_{3.6} / E_{B-V}$ and $E_{3.6-4.5} / E_{B-V}$ are often used since $E_{B-V}$ may be known a priori from other observations.

15 D. Turner (in preparation) is reworking the Galactic cluster Cepheid calibration with the aim of improving intrinsic color and distance estimates.
Those ratios are now derived from Spitzer (GLIMPSE ${ }^{16}$ ) data for O-type stars, which exhibit comparable intrinsic $(B-V)_{0}$ colors and sizable reddenings. O-type stars in the Skiff (2013) catalog were correlated with Two Micron All Sky Survey, Spitzer, and optical photometry. A linear relation between $(B-V)$ and (3.6-4.5) is displayed in Figure 2, and defines $E_{3.6-4.5} / E_{B-V}$. A robust fit applied using an iterative $\sigma$-clip yielded $E_{3.6-4.5} / E_{B-V}=0.06 \pm 0.01$.

To determine $A_{3.6} / E_{B-V}$, color ratios $\left(E_{3.6-\lambda} / E_{B-V}\right)$ were plotted as a function of $\lambda^{-1}$ and extrapolated to $\lambda \rightarrow \infty$. Intrinsic optical and near-infrared colors were adopted from Turner (1989 and references therein) and Straižys \& Lazauskaite (2009). Intrinsic mid-infrared colors were derived from the relevant color ratios (e.g., $(3.6-J)$ versus $(B-V)$ was extrapolated to the mean $(B-V)_{0}$ adopted for an O-type star). Although O-type stars are advantageous given the reasons cited above, such young stars often occupy regions displaying polycyclic aromatic hydrocarbon emission, and thus the 8.0 and $5.8 \mu \mathrm{m}$ data were bypassed to mitigate concerns regarding contamination. Figure 2 displays the extinction law diagram, whereby a polynomial fit to the data yields $A_{3.6} / E_{B-V}=$ $0.18 \pm 0.06$. The ratios derived are consistent with values adopted by Monson et al. (2012 and references therein).

Lastly, extinction laws vary throughout the Galaxy (e.g., Carraro et al. 2013; Nataf et al. 2013 and references therein), and appear anomalous for certain regions in the fourth Galactic quadrant sampled by GLIMPSE. However, altering the ratio to account for variations in the extinction law does not significantly impact estimated mid-infrared distances, hence a motivation for monitoring Cepheids with Spitzer. Applying the ratio derived

\footnotetext{
16 The Galactic Legacy Infrared Mid-Plane Survey Extraordinaire. GLIMPSE
} surveyed a portion of the fourth Galactic quadrant (Benjamin et al. 2003). 

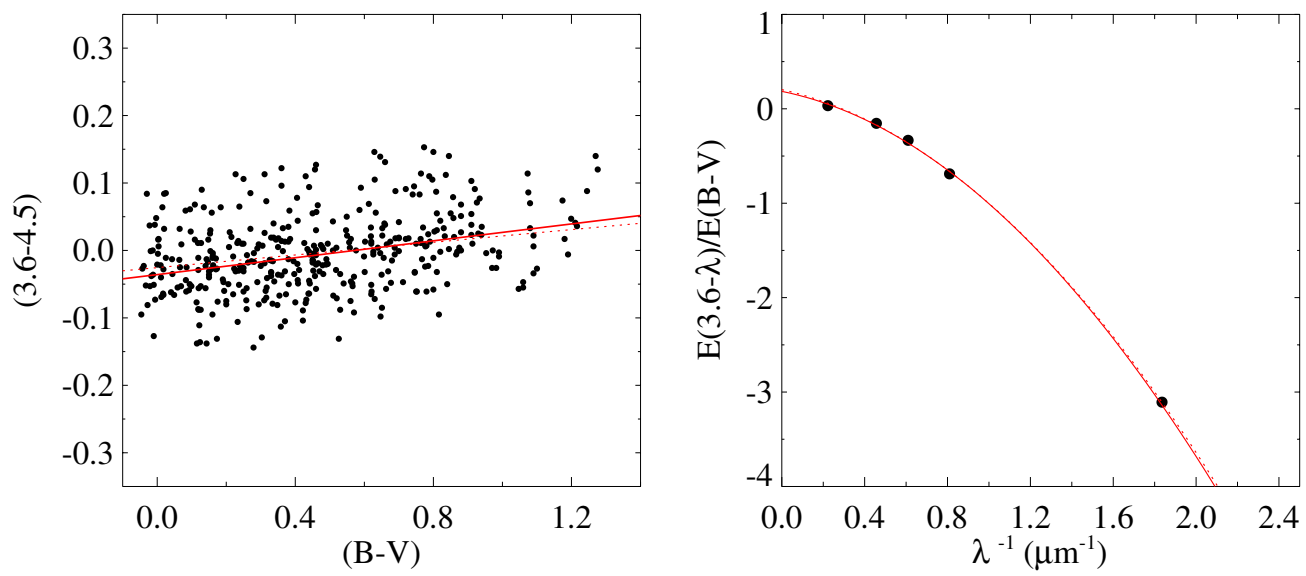

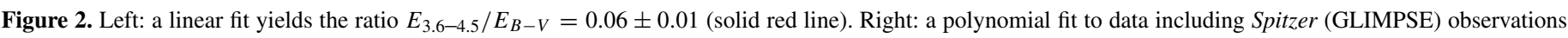

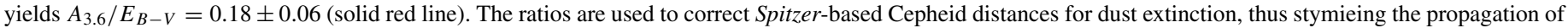
a systematic uncertainty into the distance scale. The dashed lines were inferred from parameters adopted by Monson et al. (2012 and references therein).

(A color version of this figure is available in the online journal.)

above to the mean LMC reddening $\left(E_{B-V} \sim 0.14\right)$ yields $A_{3.6} \sim 0.025$.

\subsection{Conclusions}

Spitzer 3.6 and $4.5 \mu \mathrm{m}$ period-magnitude and period-color relations (nearly mean-magnitude) were re-investigated (Figure 1). The LMC and SMC period-magnitude functions, and particularly the period-color trend, appear non-linear over an extended baseline $\left(0.45<\log P_{0}<2.0\right)$. Applying a quadratic period-magnitude function capitalizes on the statistical weight of short- and long-period Cepheids to improve certain distance determinations. Incidentally, Cepheid distances inferred from 3.6 and $4.5 \mu \mathrm{m}$ data are consistent to first order, thus indicating that $\mathrm{CO}$ features present in the $4.5 \mu \mathrm{m}$ passband do not seriously compromise the distances evaluated (the $4.5 \mu \mathrm{m}$ distances can serve as a consistency check). The slope and zero point of the relations appear comparatively insensitive to metallicity variations $\left([\mathrm{Fe} / \mathrm{H}] \sim 0\right.$ to $\left.-0.75,|\gamma|<0.1 \operatorname{mag~dex}^{-1}\right)$. That was determined in part by comparing galaxy distances $(\Delta[\mathrm{Fe} / \mathrm{H}] \sim-0.75)$ inferred from the Spitzer period-magnitude relations and NED-D. The results support prior findings (Ngeow \& Kanbur 2010; Freedman et al. 2011), yet a firm conclusion awaits a comparison between improved data for Cepheids in IC 1613 and the Milky Way. Further research is likewise required on models, as noted by Ngeow et al. (2012a), with the aim of reproducing the observed period-color trend (Figure 1). The latter is non-linear whereby (3.6-4.5) is nearly constant for shorterperiod Cepheids, and the color may transition to a bluer convex trough at longer periods (Figure 1). Lastly, an extinction law $\left(A_{3.6} / E_{B-V}=0.18 \pm 0.06\right.$, Figure 2$)$ was derived using Spitzer (GLIMPSE) observations in order to correct the distances for dust obscuration, and to avoid propagating a systematic uncertainty into the cosmic distance scale (and $H_{0}$ ).

D.M. is grateful to the following individuals and consortia whose efforts, advice, or encouragement enabled the research: Spitzer, SAGE (M. Meixner and K. Gordon), C. Ngeow, M. Marengo, H. Neilson, CHP (A. Monson, V. Scowcroft, B. Madore, and W. Freedman), S. Persson, OGLE (A. Udalski and I. Soszyñski), NED-D (I. Steer and B. Madore), GLIMPSE (R. Benjamin), B. Skiff, D. Balam, D. Lane, L. Gallo, R. Thacker, CDS (F. Ochsenbein, T. Boch, and P. Fernique),
arXiv, and NASA ADS. W.G. is grateful for support from the BASAL Centro de Astrofisica y Tecnologias Afines (CATA) PFB-06/2007.

\section{REFERENCES}

Anderson, R. I., Eyer, L., \& Mowlavi, N. 2012, arXiv:1212.5119 Becker, S. 1985, in IAU Colloq. 82, Cepheids: Theory and Observation, ed B. F. Madore (New York: Cambridge Univ. Press), 104

Benedict, G. F., McArthur, B. E., Feast, M. W., et al. 2007, AJ, 133, 1810

Benjamin, R. A., Churchwell, E., Babler, B. L., et al. 2003, PASP, 115, 953

Bono, G., Caputo, F., Marconi, M., \& Musella, I. 2010, ApJ, 715, 277

Carraro, G., Turner, D., Majaess, D., \& Baume, G. 2013, A\&A, 555, A50

Ferrarese, L., Mould, J. R., Stetson, P. B., et al. 2007, ApJ, 654, 186

Fouqué, P., Arriagada, P., Storm, J., et al. 2007, A\&A, 476, 73

Fouque, P., \& Gieren, W. P. 1997, A\&A, 320, 799

Freedman, W. L., \& Madore, B. F. 2011, ApJ, 734, 46

Freedman, W. L., Madore, B. F., Gibson, B. K., et al. 2001, ApJ, 553, 47

Freedman, W. L., Madore, B. F., Scowcroft, V., et al. 2011, AJ, 142, 192

Freedman, W. L., Madore, B. F., Scowcroft, V., et al. 2012, ApJ, 758, 24

Freedman, W. L., Rigby, J., Madore, B. F., et al. 2009, ApJ, 695, 996

Gieren, W., Pietrzyński, G., Nalewajko, K., et al. 2006, ApJ, 647, 1056

Gieren, W., Storm, J., Barnes, T. G., III, et al. 2005, ApJ, 627, 224

Gordon, K. D., Meixner, M., Meade, M. R., et al. 2011, AJ, 142, 102

Grieve, G. R., Madore, B. F., \& Welch, D. L. 1985, ApJ, 294, 513

Hackwell, J. A., \& Gehrz, R. D. 1974, ApJ, 194, 49

Luck, R. E., Moffett, T. J., Barnes, T. G., III, \& Gieren, W. P. 1998, AJ, 115,605

Macri, L. M., Stanek, K. Z., Bersier, D., Greenhill, L. J., \& Reid, M. J. 2006, ApJ, 652,1133

Madore, B. F. 1985, in IAU Colloq. 82, Cepheids: Theory and Observation, ed. B. F. Madore (New York: Cambridge Univ. Press), 166

Madore, B. F., Freedman, W. L., Rigby, J., et al. 2009, ApJ, 695, 988

Majaess, D. 2010, AcA, 60, 55

Majaess, D., Turner, D., Gieren, W., Balam, D., \& Lane, D. 2012, ApJL, 748, L9

Majaess, D., Turner, D., Gieren, W., Berdnikov, L., \& Lane, D. 2013, Ap\&SS, 344, 381

Majaess, D., Turner, D., \& Lane, D. 2009, AcA, 59, 403

Marengo, M., Evans, N. R., Barmby, P., et al. 2010, ApJ, 709, 120

Meixner, M., Gordon, K. D., Indebetouw, R., et al. 2006, AJ, 132, 2268

Monson, A. J., Freedman, W. L., Madore, B. F., et al. 2012, ApJ, 759, 146

Mottini, M., Romaniello, M., Primas, F., et al. 2006, MmSAI, 77, 156

Nataf, D. M., Gould, A., Fouqué, P., et al. 2013, ApJ, 769, 88

Neilson, H. R., Nardetto, N., Ngeow, C.-C., Fouqué, P., \& Storm, J. 2012, A\&A, 541, A134

Neilson, H. R., Ngeow, C.-C., Kanbur, S. M., \& Lester, J. B. 2009, ApJ, 692, 81

Ngeow, C., \& Kanbur, S. M. 2008, ApJ, 679, 76

Ngeow, C.-C., \& Kanbur, S. M. 2010, ApJ, 720, 626

Ngeow, C.-C., Kanbur, S. M., Bellinger, E. P., et al. 2012a, Ap\&SS, 341, 105 
Ngeow, C.-C., Marconi, M., Musella, I., Cignoni, M., \& Kanbur, S. M 2012b, ApJ, 745, 104

Ngeow, C.-C., Neilson, H. R., Nardetto, N., \& Marengo, M. 2012c, A\&A, 543, A55

Persson, S. E., Madore, B. F., Krzemiński, W., et al. 2004, AJ, 128, 2239

Pietrzyński, G., Thompson, I. B., Gieren, W., et al. 2010, Natur, 468, 542

Pietrzyński, G., Thompson, I. B., Graczyk, D., et al. 2011, ApJL, 742, L20

Planck Collaboration, Ade, P. A. R., Aghanim, N., et al. 2013, arXiv:1303.5076

Romaniello, M., Primas, F., Mottini, M., et al. 2008, A\&A, 488, 731

Sandage, A. 1988, PASP, 100, 935

Scowcroft, V., Freedman, W. L., Madore, B. F., et al. 2011, ApJ, 743, 76

Skiff, B. A. 2013, VizieR Catalog: General Catalogue of Stellar Spectral Classifications

Soszyñski, I., Poleski, R., Udalski, A., et al. 2008, AcA, 58, 163
Soszyñski, I., Poleski, R., Udalski, A., et al. 2010, AcA, 60, 17

Steer, I., \& Madore, B. 2011, NED-D: NASA/IPAC Extragalactic Database Master List of Galaxy Distances (http://ned.ipac.caltech.edu/Library/ Distances/)

Stetson, P. B., McClure, R. D., \& VandenBerg, D. A. 2004, PASP, 116,1012

Storm, J., Gieren, W., Fouqué, P., et al. 2011, A\&A, 534, A94

Straižys, V., \& Lazauskaitè, R. 2009, BaltA, 18, 19

Tammann, G. A., \& Reindl, B. 2012, Ap\&SS, 341, 3

Tammann, G. A., Sandage, A., \& Reindl, B. 2003, A\&A, 404, 423

Turner, D. G. 1989, AJ, 98, 2300

Turner, D. G. 2010, Ap\&SS, 326, 219

Turner, D. G. 2012a, Ap\&SS, 337, 303

Turner, D. G. 2012b, JAVSO, 40, 502 